Supporting Information

\title{
Total Synthesis of (-)-Melanthioidine by Copper-Mediated Cyclodimerization
}

\author{
Jianjun Wang and Gwilherm Evano* \\ Laboratoire de Chimie Organique, Service de Chimie et PhysicoChimie Organiques, Université libre de Bruxelles, \\ Avenue F. D. Roosevelt 50, CP160/06, 1050 Brussels, Belgium.
}

General Information

Experimental Procedures and Characterization Data

Assignment of the Absolute Configuration of $(R)-12$ : S14

${ }^{1} \mathrm{H}$ and ${ }^{13} \mathrm{C}$ NMR Spectra S17

Chiral HPLC Analysis of $(R)-13$ S33 


\section{General Information}

All reactions were carried out in oven-dried glassware and all solvents were reagent grade. Tetrahydrofuran was freshly distilled from sodium benzophenone. Acetonitrile, dichloromethane and 1,2-dichloroethane were freshly distilled from calcium hydride under argon. Toluene (99.8\%, Extra Dry over Molecular Sieve, AcroSeal) and $\mathrm{N}, \mathrm{N}$-dimethylformamide (99.8\%, Extra Dry, anhydrous, SC, AcroSeal) were purchased from ACROS Organics.

$[N-[(1 S, 2 S)-2-($ Amino-kN)-1,2-diphenylethyl]-4-methylbenzenesulfonamidato

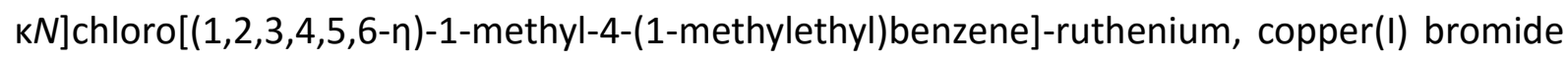
dimethylsulfide complex (99\% purity) were purchased from ACROS Organics and used as supplied. All other reagents were used as supplied.

Reactions were magnetically stirred and monitored by thin layer chromatography using Merck-Kiesegel $60 F_{254}$ plates or Macherey-Nagel Pre Coated TLC-sheets Alugram ${ }^{\circledR}$ Xtra Sil/UV 254 . Flash chromatography was performed with silica gel 60 (particle size 35-70 $\mu \mathrm{m}$ ) supplied by Merck or silica gel 60 (particle size 15-40 $\mu \mathrm{m}$ ) supplied by Macherey-Nagel. Yields refer to chromatographically and spectroscopically pure compounds unless otherwise stated.

Proton NMR spectra were recorded using an internal deuterium lock at ambient temperature on Bruker 300 and $400 \mathrm{MHz}$ spectrometers. Internal references of $\delta_{H} 7.26,3.31$, 2.05, 2.50 were used for $\mathrm{CDCl}_{3}, \mathrm{CD}_{3} \mathrm{OD}$, aceton- $d_{6}$, DMSO- $d_{6}$ respectively. Data are presented as follows: chemical shift (in ppm on the $\delta$ scale relative to $\delta_{\mathrm{TMS}}=0$ ), multiplicity (s = singlet, $\mathrm{d}$ = doublet, $\mathrm{t}=$ triplet, $\mathrm{q}=$ quartet, $\mathrm{m}=$ multiplet, $\mathrm{br}$. = broad, app. = apparent), coupling constant $(\mathrm{J} / \mathrm{Hz})$ and integration. Resonances that are either partially or fully obscured are denoted obscured (obs.). Carbon-13 NMR spectra were recorded at 75 or $100 \mathrm{MHz}$ using $\mathrm{CDCl}_{3}\left(\delta_{C}\right.$ 77.16), $\mathrm{CD}_{3} \mathrm{OD}\left(\delta_{c} 49.00\right)$, aceton- $d_{6}\left(\delta_{c} 29.84\right.$ and 206.26) and DMSO- $d_{6}\left(\delta_{c} 39.52\right)$ as internal references.

Optical rotations were recorded on an Atago AP-100 automatic polarimeter at $589 \mathrm{~nm}$ and reported as follow: $[\alpha]_{\mathrm{D}}^{25}$, concentration ( $c$ in $\mathrm{g} / 100 \mathrm{~mL}$ ), and solvent. Melting points were recorded on a Büchi Melting Point B-545. Infrared spectra were recorded on a Bruker Alpha (ATR). High-resolution mass-spectra were obtained on Agilent QTOF 6520. HPLC analysis was recorded on ThermoQuest. 


\section{Experimental Procedures and Characterization Data:}



3-(3-Bromo-4-hydroxyphenyl)propanoic acid. ${ }^{\mathrm{S1}}$ To a stirred solution of 3-(hydroxyphenyl) propionic acid ( $4.0 \mathrm{~g}, 24.1 \mathrm{mmol})$ in acetic acid $(40 \mathrm{~mL})$ was slowly added a solution of bromine $(0.6 \mathrm{~mL}, 12.1 \mathrm{mmol})$ in acetic acid $(48 \mathrm{~mL})$. The reaction mixture was then stirred for 3 hours at room temperature, diluted with ethyl acetate and washed with brine. The organic layer was dried over magnesium sulfate, filtered and concentrated under reduced pressure. The residue was finally purified by flash column chromatography over silica gel $\left(\mathrm{CHCl}_{3} / \mathrm{CH}_{3} \mathrm{OH}\right.$ : 9/1) to give the desired compound as a white solid (2.8 g, $11.2 \mathrm{mmol}, 93 \%) . \mathrm{Mp}: 78{ }^{\circ} \mathrm{C} ;{ }^{1} \mathrm{H} \mathrm{NMR}(300 \mathrm{MHz}$, $\left.\mathrm{CDCl}_{3}\right): \delta 7.31(\mathrm{~d}, J=2.1 \mathrm{~Hz}, 1 \mathrm{H}), 7.05(\mathrm{dd}, J=8.3$ and $2.1 \mathrm{~Hz}, 1 \mathrm{H}), 6.94(\mathrm{~d}, J=8.3 \mathrm{~Hz}, 1 \mathrm{H}), 2.87$ $(\mathrm{t}, J=7.7 \mathrm{~Hz}, 2 \mathrm{H}), 2.64(\mathrm{t}, J=7.7 \mathrm{~Hz}, 2 \mathrm{H}) ;{ }^{13} \mathrm{C} \mathrm{NMR}\left(\mathrm{CDCl}_{3}, 75 \mathrm{MHz}\right): \delta 179.0,150.9,133.9,131.8$, 129.2, 116.2, 110.2, 35.8, 29.5; IR (ATR): $v_{\max } 3335,1673,1509,1443,1296,1201,817,647$ $\mathrm{cm}^{-1}$;

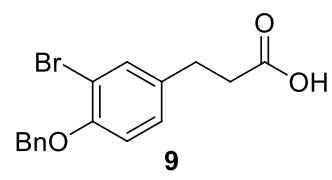

3-[4-(Benzyloxy)-3-bromophenyl]propanoic acid 9. To a solution of 3-(3-bromo-4hydroxyphenyl)propanoic acid $(4.0 \mathrm{~g}, 16.3 \mathrm{mmol})$ in acetone $(48 \mathrm{~mL})$ was added anhydrous potassium carbonate $(4.5 \mathrm{~g}, 32.7 \mathrm{mmol})$ followed by benzyl bromide $(4.3 \mathrm{~mL}, 35.9 \mathrm{mmol})$. The resulting suspension was refluxed overnight, cooled to room temperature and concentrated under reduced pressure. The residue was taken up in water and extracted with ethyl acetate. The combined organic layers were washed with brine, dried over magnesium sulfate, filtered and concentrated under reduced pressure. The crude residue was finally purified by flash

\footnotetext{
S1 This compound has been previously reported: García, J.; Franci, G.; Pereira, R.; Benedetti, R.; Nebbioso, A.;
} Rodríguez-Barrios, F.; Gronemeyer, H.; Altucci, L.; de Lera, A. R. Bioorg. Med. Chem. 2011, $19,3637$. 
column chromatography over silica gel (cyclohexane/EtOAc: 5/1) to give the corresponding benzyl 3-[4-(benzyloxy)-3-bromophenyl]propanoate as a white solid (6.6 g, $15.4 \mathrm{mmol}, 95 \%)$. To a solution of benzyl 3-[4-(benzyloxy)-3-bromophenyl]propanoate (6.6 g, $15.4 \mathrm{mmol}$ ) in THF $(17 \mathrm{~mL})$ was added a $3 \mathrm{M}$ aqueous solution of lithium hydroxide $(30.8 \mathrm{mmol}, 10.3 \mathrm{~mL})$ and methanol $(8 \mathrm{~mL})$. The reaction was stirred overnight at room temperature and the organic solvents were removed under vaccum. The residue was then acidified to $\mathrm{pH} 1-2$ by addition of a $3 \mathrm{M}$ aqueous solution of $\mathrm{HCl}$. The aqueous layer was extracted with a 1:1 mixture of ethyl acetate and dichloromethane, and the combined organic layers were concentrated under reduced pressure. The residue was dissolved in a $1 \mathrm{M}$ aqueous solution of $\mathrm{NaOH}$, and washed several times with diethyl ether. The aqueous layer was then acidified to $\mathrm{pH} 1-2$ by a $10 \%$ aqueous solution of $\mathrm{HCl}$ and extracted with ethyl acetate. The combined organic layers were washed with brine, dried over magnesium sulfate, filtered and concentrated under reduced pressure to afford the desired product as a white solid (3.2 g, $9.6 \mathrm{mmol}, 62 \%)$ Mp: $106{ }^{\circ} \mathrm{C} ;{ }^{1} \mathrm{H}$ $\operatorname{NMR}\left(\mathrm{CDCl}_{3}, 300 \mathrm{MHz}\right): \delta$ 7.48-7.32 (m, 6H), $7.07(\mathrm{dd}, J=8.4$ and $2.2 \mathrm{~Hz}, 1 \mathrm{H}), 6.86(\mathrm{~d}, J=8.4$ $\mathrm{Hz}, 1 \mathrm{H}), 5.13(\mathrm{~s}, 2 \mathrm{H}), 2.88(\mathrm{t}, J=7.6 \mathrm{~Hz}, 2 \mathrm{H}), 2.65(\mathrm{t}, J=7.6 \mathrm{~Hz}, 2 \mathrm{H}) ;{ }^{13} \mathrm{C} \mathrm{NMR}\left(\mathrm{CDCl}_{3}, 75 \mathrm{MHz}\right)$ : $\delta 178.0,153.8,136.7,134.3,133.3,128.7,128.3,128.1,127.2,114.1,112.6,71.1,35.5,29.3$; IR (ATR): $v_{\max } 2926,1714,1695,1497,1455,1290,1257,1249,1214,1048,808,737,713 \mathrm{~cm}^{-}$ 1; ESIHRMS m/z calcd for $\mathrm{C}_{16} \mathrm{H}_{19}{ }^{79} \mathrm{BrNO}_{3}\left[\mathrm{M}+\mathrm{NH}_{4}\right]^{+} 352.0543$, found 352.0535 .

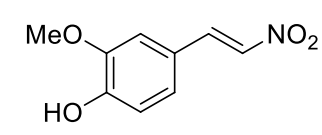

trans-4-Hydroxy-3-methoxy- $\beta$-nitrostyrene. ${ }^{\mathrm{S} 2}$ Ethylenediamine $(40 \mu \mathrm{l}, 0.6 \mathrm{mmol})$ was added to the solution of vanillin $(9.0 \mathrm{~g}, 49.2 \mathrm{mmol})$ in nitromethane $(44 \mathrm{~mL}, 819.8 \mathrm{mmol})$. The resulting solution was refluxed for 4 hours, and concentrated under vacuum. The crude crystals solid was triturated in a 1:1 methanol/ $\mathrm{H}_{2} \mathrm{O}$ mixture, collected on a Büchner funnel, rinsed with 1:1 methanol/ $\mathrm{H}_{2} \mathrm{O}$ mixture, and finally dried under vacuum to give the desired compound as a pale yellow solid $(9.5 \mathrm{~g}, 48.68 \mathrm{mmol}, 99 \%) . \mathrm{Mp}\left(\mathrm{MeOH} / \mathrm{H}_{2} \mathrm{O}\right): 143{ }^{\circ} \mathrm{C} ;{ }^{1} \mathrm{H}$ NMR (acetone- $d_{6}$,

\footnotetext{
\$2 This compound has been previously reported: Kiyokawa, K.; Nagata, T.; Hayakawa, J.; Minakata, S. Chem. Eur.
} J. 2015, 21, 1280. 
$300 \mathrm{MHz}): \delta 8.52(\mathrm{~s}, 1 \mathrm{H}), 8.02(\mathrm{~d}, J=13.4 \mathrm{~Hz}, 1 \mathrm{H}), 7.90(\mathrm{~d}, J=13.4 \mathrm{~Hz}, 1 \mathrm{H}), 7.49(\mathrm{~s}, 1 \mathrm{H}), 7.32$ (dd, $J=8.2$ and $1.2 \mathrm{~Hz}, 1 \mathrm{H}$ ), $6.92\left(\mathrm{~d}, J=8.2 \mathrm{~Hz}, 1 \mathrm{H}\right.$ ), $3.93(\mathrm{~s}, 3 \mathrm{H}) ;{ }^{13} \mathrm{C}$ NMR (acetone- $d_{6}, 75 \mathrm{MHz}$ ): $\delta 151.9,149.0,140.4,136.0,126.4,123.2,116.5,112.3,56.5$; IR (ATR): $v_{\max } 3466,1601,1518$, $1487,1474,1433,1354,1285,1246,1129,1020,952,816 \mathrm{~cm}^{-1}$.

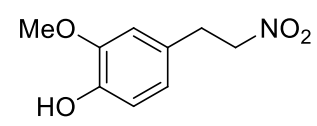

2-Methoxy-4-(2-nitroethyl)phenol. ${ }^{\mathrm{S3}}$ To a solution of trans-4-hydroxy-3-methoxy- $\beta$ nitrostyrene $(8.0 \mathrm{~g}, 41.0 \mathrm{mmol})$ in a mixture of $\mathrm{THF} / \mathrm{MeOH}(7 / 1,90 \mathrm{~mL})$ was added portionwise sodium borohydride $(4.65 \mathrm{~g}, 123.0 \mathrm{mmol})$ at $0{ }^{\circ} \mathrm{C}$. The reaction mixture was allowed to slowly warm to room temperature and stirred overnight. The reaction was quenched by slow addition of water at $0{ }^{\circ} \mathrm{C}$ and neutralized to $\mathrm{pH} 7$ by addition of a $1 \mathrm{M}$ aqueous solution of $\mathrm{HCl}$. THF was then removed under reduced pressure and the aqueous layer was extracted with diethyl ether. The combined organic layers were washed with brine, dried over magnesium sulfate, filtered and concentrated under reduced pressure. The residue was purified by column chromatography over silica gel $\left(\mathrm{CH}_{2} \mathrm{Cl}_{2} / \mathrm{MeOH}: 30 / 1\right)$ to give the desired product as a light yellow oil (7.8 g, $39.6 \mathrm{mmol}, 96 \%) .{ }^{1} \mathrm{H} \mathrm{NMR}\left(\mathrm{CDCl}_{3}, 300 \mathrm{MHz}\right): \delta 6.86(\mathrm{~d}, J=8.6 \mathrm{~Hz}, 1 \mathrm{H}), 6.69$ $6.72(\mathrm{~m}, 2 \mathrm{H}), 5.75(\mathrm{~s}, 1 \mathrm{H}), 4.58(\mathrm{t}, J=7.3 \mathrm{~Hz}, 2 \mathrm{H}), 3.88(\mathrm{~s}, 3 \mathrm{H}), 3.25(\mathrm{t}, J=7.3 \mathrm{~Hz}, 2 \mathrm{H}) ;{ }^{13} \mathrm{C} \mathrm{NMR}$ $\left(\mathrm{CDCl}_{3}, 75 \mathrm{MHz}\right): \delta 146.8,144.9,128.0,121.3,114.8,111.2,76.7,56.0,33.2 ;$ IR (ATR): $v_{\max } 3509$, $1539,1515,1429,1269,1238,1212,1147,1024,904,850,790 \mathrm{~cm}^{-1}$.



4-Hydroxy-3-methoxy-phenethylamine hydrochloride 7. To a solution of 2-methoxy-4-(2nitroethyl)phenol $(7.8 \mathrm{~g}, 39.6 \mathrm{mmol})$ in ethanol $(120 \mathrm{~mL})$, was added palladium on carbon $(10 \%$ wt., $780 \mathrm{mg}$ ) and the reaction mixture was stirred at room temperature overnight under an

\footnotetext{
S3 This compound has been previously reported: Zhang, Z.; Schreiner, P. R. Synthesis 2007, 16, 2559.
} 
hydrogen atmosphere (2.0 bars). The reaction mixture was then filtered on a Büchner funnel and the filtrate was then concentrated under reduced pressure and co-evaporated with ethyl acetate. The residue was then dissolved in a $1 \mathrm{M}$ aqueous solution of $\mathrm{HCl}$ and washed several times with diethyl ether. The aqueous phase was finally concentrated under reduced pressure to give the desired product as a brown solid (2.5 g, $12.2 \mathrm{mmol}, 31 \%)$. Mp: $179{ }^{\circ} \mathrm{C} ;{ }^{1} \mathrm{H} \mathrm{NMR}$ $\left(\mathrm{CD}_{3} \mathrm{OD}, 300 \mathrm{MHz}\right): \delta 6.98(\mathrm{~d}, J=1.9 \mathrm{~Hz}, 1 \mathrm{H}), 6.91(\mathrm{~d}, J=8.0 \mathrm{~Hz}, 1 \mathrm{H}), 6.82(\mathrm{dd}, J=8.0$ and 1.9 $\mathrm{Hz}, 1 \mathrm{H}), 3.87(\mathrm{~s}, 3 \mathrm{H}), 3.25(\mathrm{t}, J=7.4 \mathrm{~Hz}, 2 \mathrm{H}), 2.93(\mathrm{t}, J=7.4 \mathrm{~Hz}, 2 \mathrm{H}) ;{ }^{13} \mathrm{C} \mathrm{NMR}\left(\mathrm{CD}_{3} \mathrm{OD}, 75 \mathrm{MHz}\right)$ : $\delta 149.3,146.8,129.1,122.3,116.6,113.4,56.4,42.2,34.1$; IR (ATR): $v_{\max } 3130,1600,1526$, $1483,1279,1251,1213,1158,1123,1035,819 \mathrm{~cm}^{-1}$; ESIHRMS m/z calcd for $\mathrm{C}_{9} \mathrm{H}_{14} \mathrm{NO}_{2}[\mathrm{M}-\mathrm{Cl}]^{+}$ 168.1019, found 168.1015 .

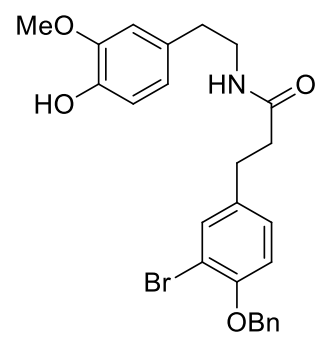

N-(4-Hydroxy-3-methoxyphenethyl)-3-(4'-benzyloxy-3'-bromophenyl)propionamide. To a solution of 9 (3.3 g, $9.9 \mathrm{mmol})$ and $7(2.0 \mathrm{~g}, 9.9 \mathrm{mmol})$ in dry DMF (34 mL) was added 1hydroxybenzotriazole $(1.6 \mathrm{~g}, 11.5 \mathrm{mmol})$. The reaction mixture was then cooled to $0{ }^{\circ} \mathrm{C}$ and 1 (3-dimethylaminopropyl)-3-ethyl-carbodiimide hydrochloride (1.9 g, $9.9 \mathrm{mmol})$ and $\mathrm{N}$ methylmorpholine $(3.1 \mathrm{~mL}, 27.7 \mathrm{mmol})$ were then added. The reaction mixture was slowly warm to room temperature and stirred for 24 hours before being quenched by addition of a saturated aqueous solution of sodium bicarbonate and extracted with ethyl acetate. The combined organic layers were washed with brine, dried over magnesium sulfate, filtered and concentrated under reduced pressure. The residue was purified by flash chromatography over silica gel (EtOAc/cyclohexane: 2/1) to give the desired compound as a white solid (3.3 g, 6.8 mmol, 69\%). Mp: $95{ }^{\circ} \mathrm{C} ;{ }^{1} \mathrm{H} \mathrm{NMR}\left(\mathrm{CDCl}_{3}, 300 \mathrm{MHz}\right): \delta 7.47-7.45(\mathrm{~m}, 2 \mathrm{H}), 7.40-7.30(\mathrm{~m}, 4 \mathrm{H}), 7.03$ (dd, $J=8.4$ and $2.1 \mathrm{~Hz}, 1 \mathrm{H}), 6.84(\mathrm{~s}, 1 \mathrm{H}), 6.81(\mathrm{~s}, 1 \mathrm{H}), 6.65(\mathrm{~d}, J=1.8 \mathrm{~Hz}, 1 \mathrm{H}), 6.57(\mathrm{dd}, J=8.0$ and $1.8 \mathrm{~Hz}, 1 \mathrm{H}$ ), $5.57(\mathrm{~s}, 1 \mathrm{H}$ ), 5.36 (br. t, $J=4.6 \mathrm{~Hz}, 1 \mathrm{H}$ ), 5.12 (s, 2H), 3.85 (s, 3H), 3.44 (app. q, 
$J=6.9 \mathrm{~Hz}, 2 \mathrm{H}), 2.80(\mathrm{t}, J=7.6 \mathrm{~Hz}, 2 \mathrm{H}), 2.67(\mathrm{t}, J=6.9 \mathrm{~Hz}, 2 \mathrm{H}), 2.37(\mathrm{t}, J=7.6 \mathrm{~Hz}, 2 \mathrm{H}) ;{ }^{13} \mathrm{C} \mathrm{NMR}$ $\left(\mathrm{CDCl}_{3}, 75 \mathrm{MHz}\right): \delta 171.8,153.6,146.8,144.4,136.7,135.0,133.3,130.7,128.7,128.5,128.1$, 127.1, 121.4, 114.5, 114.1, 112.6, 111.2, 71.1, 56.1, 40.8, 38.6, 35.5, 30.6; IR (ATR): $v_{\max } 3299$, 1643, 1556, 1518, 1499, 1268, 1234, 1054, 1029, 849, $733 \mathrm{~cm}^{-1}$; ESIHRMS m/z calcd for $\mathrm{C}_{25} \mathrm{H}_{27}{ }^{81} \mathrm{BrNO}_{4}[\mathrm{M}+\mathrm{H}]^{+}$486.1098, found 486.1105.



$\mathbf{N}$-(4-Acetoxy-3-methoxyphenethyl)-3-(4'-benzyloxy-3'-bromophenyl)propionamide 5. To a solution of $\quad N$-(4-hydroxy-3-methoxyphenethyl)-3-(4'-benzyloxy-3'-bromophenyl) propionamide $(200 \mathrm{mg}, 0.43 \mathrm{mmol})$ in pyridine $(8 \mathrm{~mL})$ was added acetic anhydride $(9.6 \mathrm{~mL}$, $102 \mathrm{mmol}$ ) at room temperature and stirred overnight. The reaction mixture was then cooled to $0{ }^{\circ} \mathrm{C}$, quenched by slowly addition of water at $0{ }^{\circ} \mathrm{C}$ and extracted with ethyl acetate. The combined organic layers were washed with brine, dried over magnesium sulfate, concentrated under reduced pressure and co-evaporated with toluene to give the desired product as a white solid (212 mg, $0.40 \mathrm{mmol}, 98 \%)$. Mp: $92{ }^{\circ} \mathrm{C} ;{ }^{1} \mathrm{H} \mathrm{NMR}\left(\mathrm{CDCl}_{3}, 300 \mathrm{MHz}\right): \delta$ 7.47-7.45 (m, 2H), 7.40-7.30 (m, 4H), $7.06(\mathrm{dd}, J=8.4$ and $2.0 \mathrm{~Hz}, 1 \mathrm{H}), 6.95(\mathrm{~d}, J=8.0 \mathrm{~Hz}, 1 \mathrm{H}), 6.85(\mathrm{~d}, J=8.4 \mathrm{~Hz}$, 1H), $6.76(\mathrm{~d}, J=1.7 \mathrm{~Hz}, 1 \mathrm{H}), 6.65(\mathrm{dd}, J=8.0$ and $1.7 \mathrm{~Hz}, 1 \mathrm{H}$ ), 5.46 (br. t, $J=5.3 \mathrm{~Hz}, 1 \mathrm{H}), 5.14$ (s, $2 \mathrm{H}), 3.80(\mathrm{~s}, 3 \mathrm{H}), 3.47$ (app. q, $J=6.8 \mathrm{~Hz}, 2 \mathrm{H}), 2.88(\mathrm{t}, J=7.4 \mathrm{~Hz}, 2 \mathrm{H}), 2.74(\mathrm{t}, J=6.8 \mathrm{~Hz}, 2 \mathrm{H})$, $2.39(\mathrm{t}, J=7.4 \mathrm{~Hz}, 2 \mathrm{H}), 2.32(\mathrm{~s}, 3 \mathrm{H}) ;{ }^{13} \mathrm{C} \mathrm{NMR}\left(\mathrm{CDCl}_{3}, 75 \mathrm{MHz}\right): \delta 171.9,169.4,153.6,151.2$, 138.5, 137.9, 136.7, 135.0, 133.3, 128.7, 128.5, 128.0, 127.1, 122.9, 120.9, 114.1, 112.9, 112.5, 71.0, 56.0, 40.6, 38.5, 35.8, 30.6, 20.8; IR (ATR): $v_{\max } 3307,2927,1756,1640,1557,1518,1258$, 1227, 1203, 1030, 826, $738 \mathrm{~cm}^{-1}$; ESIHRMS m/z calcd for $\mathrm{C}_{27} \mathrm{H}_{29}{ }^{81} \mathrm{BrNO}_{5}[\mathrm{M}+\mathrm{H}]^{+} 528.1203$, found 528.1208 . 


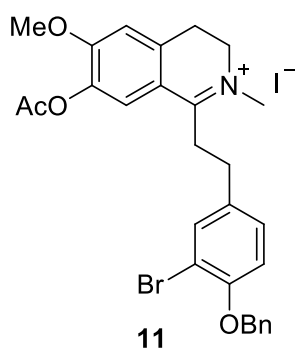

\section{7-Acetoxy-1-[4-(benzyloxy)-3-bromophenethyl]-6-methoxy-2-methyl-3,4-}

dihydroisoquinoline-2-ium iodide 11 . To a solution of 5 (600 mg, $1.14 \mathrm{mmol})$ in dry toluene $(12 \mathrm{~mL})$ was added phosphoryl chloride $(400 \mu \mathrm{l}, 4.3 \mathrm{mmol})$. The reaction mixture was refluxed for 4 hours and concentrated under reduced pressure. The residue was then dissolved in ethyl acetate $(9 \mathrm{~mL})$ and stirred one hour under argon along with a $10 \%$ aqueous solution of potassium carbonate $(27 \mathrm{~mL})$. The organic layer was separated, washed with brine, dried over magnesium sulfate and concentrated. lodomethane $(1.0 \mathrm{ml}, 16.0 \mathrm{mmol})$ and dry diethyl ether $(8 \mathrm{~mL})$ were then added to the residue until precipitation occurred and the mixture was kept at $4{ }^{\circ} \mathrm{C}$ overnight. The precipitate was collected by filtration on a Büchner funnel and the filtrate was concentrated and treated with iodomethane and dry diethyl ether two times more to collect all the desired product as a yellow solid (550 mg, $0.85 \mathrm{mmol}, 74 \%$ ). Mp: $166{ }^{\circ} \mathrm{C} ;{ }^{1} \mathrm{H}$ NMR (DMSO- $d_{6}, 400 \mathrm{MHz}$ ): $\delta 7.87(\mathrm{~s}, 1 \mathrm{H}), 7.56(\mathrm{~d}, J=2.0 \mathrm{~Hz}, 1 \mathrm{H}), 7.47-7.45(\mathrm{~m}, 2 \mathrm{H}), 7.43-7.39$ $(\mathrm{m}, 2 \mathrm{H}), 7.36-7.33(\mathrm{~m}, 1 \mathrm{H}), 7.29(\mathrm{~s}, 1 \mathrm{H}), 7.20(\mathrm{dd}, J=8.5$ and $2.0 \mathrm{~Hz}, 1 \mathrm{H}), 7.09(\mathrm{~d}, J=8.5 \mathrm{~Hz}$, 1H), $5.15(\mathrm{~s}, 2 \mathrm{H}), 3.99(\mathrm{t}, J=7.6 \mathrm{~Hz}, 2 \mathrm{H}), 3.92(\mathrm{~s}, 3 \mathrm{H}), 3.66(\mathrm{~s}, 3 \mathrm{H}), 3.41(\mathrm{t}, J=7.4 \mathrm{~Hz}, 2 \mathrm{H}), 3.08$ (t, $J=7.6 \mathrm{~Hz}, 2 \mathrm{H}), 2.83(\mathrm{t}, J=7.4 \mathrm{~Hz}, 2 \mathrm{H}), 2.30$ (s, 3H); ${ }^{13} \mathrm{C}$ NMR (DMSO-d $6,100 \mathrm{MHz}$ ): $\delta$ 175.0, $168.3,157.0,153.2,138.4$ (2C), 136.6, 133.0, 132.7, 129.1, 128.5, 127.9 127.3, 125.3, 118.7, $114.2,112.3,111.2,70.0,56.8,52.0,44.1,31.5,31.1,25.1,20.3$; IR (ATR): $v_{\max } 3011,1756$, $1607,1566,1498,1455,1378,1299,1264,1219,1145,1023,804,740,696 \mathrm{~cm}^{-1}$; ESIHRMS $\mathrm{m} / \mathrm{z}$ calcd $\mathrm{C}_{28} \mathrm{H}_{29}{ }^{81} \mathrm{BrNO}_{4}[\mathrm{M}-\mathrm{I}]^{+}$524.1254, found 524.1254. 


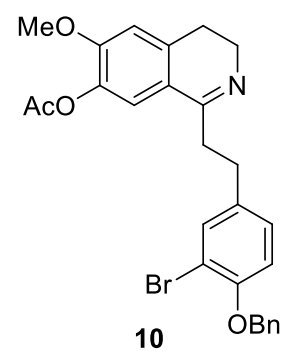

7-Acetoxy-6-methoxy-1-(3'-bromo-4'-benzyloxyphenethyl)-3,4-dihydroisoquinoline

10.

Phosphoryl chloride (396 $\mu \mathrm{l}, 4.25 \mathrm{mmol})$ was added to a solution of 5 (700 mg, $1.33 \mathrm{mmol}$ ) in dry acetonitrile $(5 \mathrm{~mL})$ at $60^{\circ} \mathrm{C}$. The reaction mixture was then refluxed for 4 hours, cooled to room temperature and concentrated under reduced pressure. The residue was treated with a saturated aqueous solution of sodium bicarbonate and the aqueous layer was extracted with dichloromethane. The combined organic layer was washed with brine, dried over magnesium sulfate, filtered and concentrated under reduced pressure to give the desired product as a yellowish gummy product (608 $\mathrm{mg}, 1.20 \mathrm{mmol}, 90 \%)$, which was directly used in the next step without further purification. ${ }^{1} \mathrm{H}$ NMR $\left(\mathrm{CDCl}_{3}, 300 \mathrm{MHz}\right): \delta$ 7.48-7.46 $(\mathrm{m}, 2 \mathrm{H}), 7.41-7.29(\mathrm{~m}, 4 \mathrm{H})$, $7.13(\mathrm{~s}, 1 \mathrm{H}), 7.07(\mathrm{dd}, J=8.3$ and $2.0 \mathrm{~Hz}, 1 \mathrm{H}), 6.84(\mathrm{~d}, J=8.3 \mathrm{~Hz}, 1 \mathrm{H}), 6.78(\mathrm{~s}, 1 \mathrm{H}), 5.13(\mathrm{~s}, 2 \mathrm{H})$, $3.87(\mathrm{~s}, 3 \mathrm{H}), 3.67(\mathrm{t}, J=7.6 \mathrm{~Hz}, 2 \mathrm{H}), 2.90$ (app. s, 4H), $2.66(\mathrm{t}, J=7.6 \mathrm{~Hz}, 2 \mathrm{H}), 2.32(\mathrm{~s}, 3 \mathrm{H}) ;{ }^{13} \mathrm{C}$ $\operatorname{NMR}\left(\mathrm{CDCl}_{3}, 75 \mathrm{MHz}\right): \delta$ 169.1, 165.3, 153.3, 152.8, 138.1, 137.4, 136.8, 135.9, 133.3, 128.6, 128.4, 127.9, 127.1, 122.0, 119.9, 114.1, 112.3, 111.4, 71.0, 56.1, 46.6, 37.3, 31.8, 26.3, 20.7; IR (ATR): $v_{\max } 2934,1764,1495,1316,1204,1135,1049,738,697 \mathrm{~cm}^{-1}$; ESIHRMS m/z calcd $\mathrm{C}_{27} \mathrm{H}_{27}{ }^{81} \mathrm{BrNO}_{4}[\mathrm{M}+\mathrm{H}]^{+}$510.1098, found 510.1087.



(R)-7-Acetoxy-6-methoxy-1-(3'-bromo-4'-benzyloxyphenethyl)-1,2,3,4-

tetrahydroisoquinoline hydrochloride 12. To a solution of $10(1.2 \mathrm{~g}, 2.40 \mathrm{mmol})$ in dry DMF $(40 \mathrm{~mL})$ was added RuCl[(S,S)-TsDPEN](p-cymene) $(31 \mathrm{mg}, 0.10 \mathrm{mmol})$ at room temperature. 
The mixture was then cooled to $0{ }^{\circ} \mathrm{C}$ before the addition of triethylamine $(1.2 \mathrm{~mL}, 8.6 \mathrm{mmol})$ and formic acid $(2.9 \mathrm{~mL}, 73.8 \mathrm{mmol})$ under argon. The reaction was then allowed to warm to room temperature and stirred for two days at room temperature before being quenched by addition of a saturated aqueous solution of sodium bicarbonate and being extracted with dichloromethane. The combined organic layers were washed with brine, dried over magnesium sulfate, filtered and concentrated under reduced pressure. The residue was dissolved in a $0.48 \mathrm{M}$ solution of $\mathrm{HCl}$ in diethyl ether $(5 \mathrm{~mL}, 2.5 \mathrm{mmol})$, stirred for 5 minutes and then concentrated under reduced pressure. This last step was repeated three times to give the desired product as a brownish solid (1.3 g, $2.3 \mathrm{mmol}, 95 \%)$. Mp: $140{ }^{\circ} \mathrm{C} ;[\alpha]_{\mathrm{D}}^{25}-15.0$ (c 0.28 , $\left.\mathrm{CHCl}_{3}\right) ;{ }^{1} \mathrm{H} \mathrm{NMR}\left(\mathrm{CD}_{3} \mathrm{OD}, 300 \mathrm{MHz}\right): \delta 7.49-7.45(\mathrm{~m}, 3 \mathrm{H}), 7.39-7.29(\mathrm{~m}, 3 \mathrm{H}), 7.18(\mathrm{dd}, J=8.4$ and $2.1 \mathrm{~Hz}, 1 \mathrm{H}), 7.02(\mathrm{~d}, J=8.4 \mathrm{~Hz}, 1 \mathrm{H}), 6.99(\mathrm{~s}, 1 \mathrm{H}), 6.96$ (s, 1H), 5.14 (s, 2H), 4.50 (dd, J = 7.5 and $5.0 \mathrm{~Hz}, 1 \mathrm{H}), 3.82(\mathrm{~s}, 3 \mathrm{H}), 3.66-3.58(\mathrm{~m}, 1 \mathrm{H}), 3.46-3.38(\mathrm{~m}, 1 \mathrm{H}), 3.24-3.05(\mathrm{~m}, 2 \mathrm{H}), 2.79-2.67(\mathrm{~m}$, 2H), 2.35-2.17 (m, 5H); $\left.{ }^{13} \mathrm{C} \mathrm{NMR} \mathrm{(CD} \mathrm{OD}, 75 \mathrm{MHz}\right): \delta 170.6,155.0,152.5,140.3,138.2,135.7$, 134.2, 131.7, 129.5, 129.4, 128.9, 128.3, 125.1, 122.1, 115.4, 113.8, 113.3, 71.9, 56.5, 55.9, 40.7, 36.8, 31.2, 26.2, 20.4; IR (ATR): $V_{\max } 2940,1761,1515,1496,1258,1197,1116,1014,909$, $813 \mathrm{~cm}^{-1} ;$ ESIHRMS m/z calcd $\mathrm{C}_{27} \mathrm{H}_{29}{ }^{81} \mathrm{BrNO}_{4}[\mathrm{M}-\mathrm{Cl}]^{+}$512.1254, found 512.1257.



\section{(R)-7-Acetoxy-6-methoxy-1-(3'-bromo-4'-benzyloxyphenethyl)- $\mathrm{N}$-methyl-1,2,3,4-}

tetrahydroisoquinoline 13. To a suspension of 12 (1.3 g, $2.2 \mathrm{mmol})$ in dry 1,2-dichloroethane $(38 \mathrm{~mL})$ was added $N, N$-diisopropylethylamine $(488 \mu \mathrm{l}, 2.8 \mathrm{mmol})$ and the suspension was vigorously stirred at $0{ }^{\circ} \mathrm{C}$ for $30 \mathrm{~min}$ before addition of an aqueous solution of formaldehyde (37\% wt., $1.7 \mathrm{~mL}, 22.8 \mathrm{mmol})$. The reaction mixture was then slowly warmed to room temperature, stirred one hour and then cooled back to $0{ }^{\circ} \mathrm{C}$. Sodium triacetoxyborohydride $(2.6 \mathrm{~g}, 12.0 \mathrm{mmol})$ was then added portionwise and the reaction mixture was allowed to warm 
to room temperature and stirred for 16 hours. The reaction mixture was then cooled to $0{ }^{\circ} \mathrm{C}$ and quenched by addition of water before being diluted with dichloromethane. The aqueous layer was basified to $\mathrm{pH}$ 9-10 by addition of a $1 \mathrm{M}$ aqueous solution of $\mathrm{NaOH}$ and extracted with dichloromethane. The combined organic layers were washed with brine, dried over magnesium sulfate, filtered and concentrated under reduced pressure. The residue was purified by flash chromatography over silica gel (EtOAc/MeOH: 8/1) to give the desired product as a yellow gummy product (949 mg, $1.8 \mathrm{mmol}, 79 \%)$; $[\alpha]_{\mathrm{D}}^{25}+34.5\left(c 0.11, \mathrm{CHCl}_{3}\right) ; 91 \%$ ee $(R)$. The ee value was determined by comparison of HPLC analyses of the optically enriched product and the racemic one obtained by reduction of $\mathbf{1 1}$ with ( $p$-cymene)ruthenium(II) chloride dimer (Daicel Chiralpak IA-3, n-hexane/propan-2-ol =95/5, flow rate $=1.0 \mathrm{~mL} \cdot \mathrm{min}^{-1}$, $\left.210 \mathrm{~nm}, t_{S}=5.75 \mathrm{~min}(S), t_{R}=7.04(R)\right) ;{ }^{1} \mathrm{H} \mathrm{NMR}\left(\mathrm{CDCl}_{3}, 300 \mathrm{MHz}\right): \delta 7.48-7.46(\mathrm{~m}, 2 \mathrm{H}), 7.41-$ $7.29(\mathrm{~m}, 4 \mathrm{H}), 7.02(\mathrm{dd}, J=8.3$ and $1.6 \mathrm{~Hz}, 1 \mathrm{H}), 6.83(\mathrm{~d}, J=8.3 \mathrm{~Hz}, 1 \mathrm{H}), 6.74(\mathrm{~s}, 1 \mathrm{H}), 6.66(\mathrm{~s}, 1 \mathrm{H})$, $5.12(\mathrm{~s}, 2 \mathrm{H}), 3.80(\mathrm{~s}, 3 \mathrm{H}), 3.39$ (br. t, $J=4.8 \mathrm{~Hz}, 1 \mathrm{H}), 3.16-3.09(\mathrm{~m}, 1 \mathrm{H}), 2.78-2.63(\mathrm{~m}, 4 \mathrm{H}), 2.45$

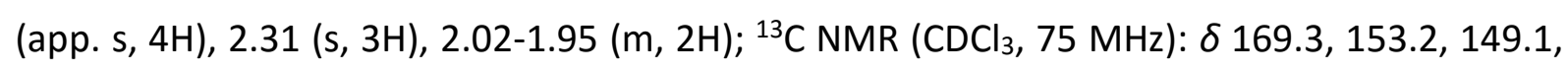
138.0, 137.1, 136.9, 133.4 (2C), 130.1, 128.7, 128.4, 128.0, 127.2, 121.2, 114.1, 112.4 (2C), 71.1, 62.3, 57.0, 48.4, 42.7, 36.9, 30.2, 26.2, 20.8 ; IR (ATR): $v_{\max } 2931,1763,1511,1454,1370$, 1260, 1205, 1189, 1018, 910, 808, $735 \mathrm{~cm}^{-1}$; ESIHRMS m/z calcd $\mathrm{C}_{28} \mathrm{H}_{31}{ }^{79} \mathrm{BrNO}_{4}[\mathrm{M}+\mathrm{H}]^{+}$ 524.1431, found 524.1430.

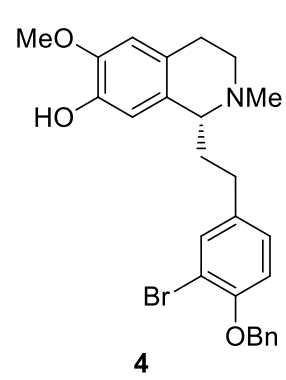

(R)-7-Hydroxy-6-methoxy-1-(3'-bromo-4'-benzyloxyphenethyl)-N-methyl-1,2,3,4-

tetrahydroisoquinoline 4. Potassium carbonate $(1.0 \mathrm{~g}, 7.2 \mathrm{mmol})$ was added to a solution of 13 (650 mg, $1.24 \mathrm{mmol})$ in methanol $(40 \mathrm{~mL})$ and the mixture was stirred overnight at room temperature. The reaction mixture was then acidified by addition of a $1 \mathrm{M}$ aqueous solution of $\mathrm{HCl}$ to $\mathrm{pH} 4-5$ and extracted with ethyl acetate. The combined organic layer were washed with 
brine, dried over magnesium sulfate, filtered and concentrated under reduced pressure. The residue was purified by flash chromatography over silica gel $\left(\mathrm{CH}_{2} \mathrm{Cl}_{2} / \mathrm{MeOH}: 10 / 1\right)$ to give the desired product as a yellowish solid (558 mg, $1.16 \mathrm{mmol}, 93 \%)$. Mp: $89^{\circ} \mathrm{C}$; $[\alpha]_{\mathrm{D}}^{25}-21.3$ (c 0.15 , $\left.\mathrm{CHCl}_{3}\right) ;{ }^{1} \mathrm{H} \mathrm{NMR}\left(\mathrm{CDCl}_{3}, 400 \mathrm{MHz}\right): \delta$ 7.48-7.46 (m, 2H), 7.40-7.36 (m, 3H), 7.33-7.29 (m, $\left.1 \mathrm{H}\right)$, $7.03(\mathrm{dd}, J=6.3$ and $1.5 \mathrm{~Hz}, 1 \mathrm{H}), 6.83(\mathrm{~d}, J=6.3 \mathrm{~Hz}, 1 \mathrm{H}), 6.64(\mathrm{~s}, 1 \mathrm{H}), 6.55(\mathrm{~s}, 1 \mathrm{H}), 5.12(\mathrm{~s}, 2 \mathrm{H})$, $3.84(\mathrm{~s}, 3 \mathrm{H}), 3.39(\mathrm{t}, J=3.8 \mathrm{~Hz}, 1 \mathrm{H}), 3.17-3.09(\mathrm{~m}, 1 \mathrm{H}), 2.78-2.62(\mathrm{~m}, 4 \mathrm{H}), 2.47$ (app. s, 4H), 2.02-1.97 (m, 2H); ${ }^{13} \mathrm{CNMR}\left(\mathrm{CDCl}_{3}, 100 \mathrm{MHz}\right): \delta 153.1,145.1,144.0,137.2,137.0,133.4,130.5$, 128.6, 128.4, 128.0, 127.2, 126.2, 114.1, 112.9, 112.4, 110.7, 71.1, 62.6, 56.0, 48.6, 42.8, 36.7, 30.3, 25.9; IR (ATR): $v_{\max } 2931,1512,1495,1454,1274,1253,1050,1022,737,696 \mathrm{~cm}^{-1}$; ESIHRMS m/z calcd $\mathrm{C}_{26} \mathrm{H}_{29}{ }^{79} \mathrm{BrNO}_{3}[\mathrm{M}+\mathrm{H}]^{+}$482.1325, found 482.1320.



14

(-)-O,O-Dibenzyl-melanthioidine 14. A $10 \mathrm{~mL}$ pressure tube was charged with 4 (80 mg, 0.166 mmol), copper(l) bromide dimethyl sulfide complex (45 mg, $0.216 \mathrm{mmol}$ ) and cesium carbonate (162 $\mathrm{mg}, 0.498 \mathrm{mmol})$. The tube was fitted with a rubber septum, evacuated under high vacuum and backfilled with argon. Freshly distilled pyridine $(1.0 \mathrm{~mL})$ was then added and the rubber septum was replaced by a Teflon-coated screw cap. The resulting mixture was heated to $150{ }^{\circ} \mathrm{C}$ for 48 hours, concentrated under reduced pressure, diluted with ethyl acetate and then filtered through a small plug of silica gel. The filtrate was concentrated under reduced pressure and finally purified by flash chromatography over silica gel (chloroform/methanol: 10/1) to give the desired product as a brown solid ( $24 \mathrm{mg}, 0.029 \mathrm{mmol}$, 35\%). Mp: $93{ }^{\circ} \mathrm{C} ;[\alpha]_{\mathrm{D}}^{25}-32.1$ (c 0.35, $\left.\mathrm{CHCl}_{3}\right) ;{ }^{1} \mathrm{H} \mathrm{NMR}\left(\mathrm{CDCl}_{3}, 300 \mathrm{MHz}\right): \delta$ 7.35-7.26 (m, 10H), $6.87(\mathrm{~d}, J=8.3 \mathrm{~Hz}, 2 \mathrm{H}), 6.78(\mathrm{dd}, J=8.3$ and $1.4 \mathrm{~Hz}, 2 \mathrm{H}), 6.67(\mathrm{~s}, 2 \mathrm{H}), 6.52(\mathrm{~d}, J=1.4 \mathrm{~Hz}, 2 \mathrm{H}$ ), $6.47(\mathrm{~s}, 2 \mathrm{H}$ ), 5.16 ( $\mathrm{A}$ of $A B$ syst. $J=12.4 \mathrm{~Hz}, 2 \mathrm{H}$ ), 5.09 (B of AB syst. $J=12.4 \mathrm{~Hz}, 2 \mathrm{H}$ ), $3.80(\mathrm{~s}, 6 \mathrm{H}$ ), 
$3.36(\mathrm{t}, J=5.0 \mathrm{~Hz}, 2 \mathrm{H}), 3.10-3.04(\mathrm{~m}, 2 \mathrm{H}), 2.79-2.66(\mathrm{~m}, 6 \mathrm{H}), 2.59-2.49(\mathrm{~m}, 8 \mathrm{H}), 2.26-2.17(\mathrm{~m}$,

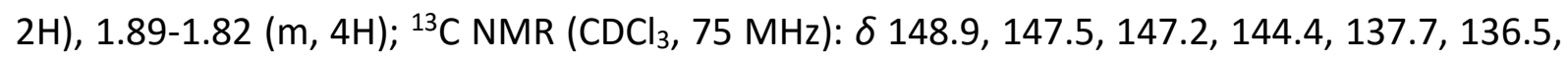
130.0, 129.9, 128.5, 127.7, 127.4, 123.0, 118.9, 117.7, 116.2, 112.9, 71.6, 62.6, 56.2, 48.4, 42.8, 36.1, 30.8, 26.5. IR (ATR): $v_{\max } 2854,1611,1509,1266,1214,1096,1023,735 \mathrm{~cm}^{-1}$; ESIHRMS $\mathrm{m} / \mathrm{z}$ calcd $\mathrm{C}_{52} \mathrm{H}_{55} \mathrm{~N}_{2} \mathrm{O}_{6}[\mathrm{M}+\mathrm{H}]^{+} 803.4055$, found 803.4043 .

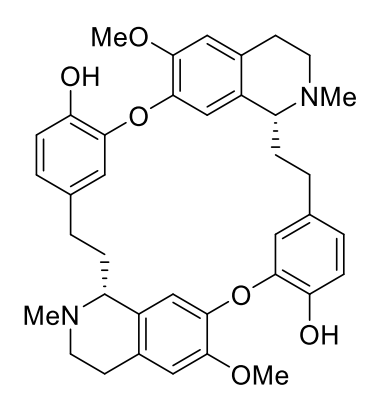

3

(-)-Melanthioidine 3. To a solution of $14(34 \mathrm{mg}, 0.042 \mathrm{mmol})$ in ethanol ( $8 \mathrm{~mL}$ ) was added a $37 \%$ aqueous solution of $\mathrm{HCl}(4.4 \mathrm{~mL})$ and the resulting mixture was refluxed for 5 hours. The reaction mixture was then cooled to $0{ }^{\circ} \mathrm{C}$ and basified by a $25 \%$ aqueous solution of ammonia. The aqueous layer was extracted with dichloromethane and the combined organic layers were washed with brine, dried over magnesium sulfate, filtered and concentrated under reduced pressure. The crude product was finally purified by flash chromatography over silica gel (chloroform/methanol: 9/1) to give the desired product as a white solid ( $23 \mathrm{mg}, 0.037 \mathrm{mmol}$, 88\%). Mp (ACOEt): $136-137^{\circ} \mathrm{C} ;[\alpha]_{\mathrm{D}}^{25}-147.9$ (c 0.12, $\left.\mathrm{CHCl}_{3}\right) ;{ }^{1} \mathrm{H} \mathrm{NMR}\left(\mathrm{CDCl}_{3}, 300 \mathrm{MHz}\right): \delta 6.91$ $(\mathrm{d}, J=8.2 \mathrm{~Hz}, 2 \mathrm{H}), 6.83(\mathrm{dd}, J=8.2$ and $1.9 \mathrm{~Hz}, 2 \mathrm{H}), 6.71(\mathrm{~s}, 2 \mathrm{H}), 6.66(\mathrm{~s}, 2 \mathrm{H}), 6.56(\mathrm{~d}, J=1.9 \mathrm{~Hz}$, $2 \mathrm{H}), 3.79(\mathrm{~s}, 6 \mathrm{H}), 3.40(\mathrm{t}, J=5.6 \mathrm{~Hz}, 2 \mathrm{H}), 3.16-3.07(\mathrm{~m}, 2 \mathrm{H}), 2.88-2.66(\mathrm{~m}, 6 \mathrm{H}), 2.62-2.52(\mathrm{~m}$, $2 \mathrm{H}), 2.46(\mathrm{~s}, 6 \mathrm{H}), 2.39-2.27(\mathrm{~m}, 2 \mathrm{H}), 2.03-1.91(\mathrm{~m}, 2 \mathrm{H}), 1.87-1.75(\mathrm{~m}, 2 \mathrm{H}) ;{ }^{13} \mathrm{C} \mathrm{NMR}\left(\mathrm{CDCl}_{3}, 75\right.$ $\mathrm{MHz}): \delta 149.2,145.3,144.9,143.6,134.6,131.0,130.3,124.0,119.4,117.3,116.0,112.9,62.4$, 56.1, 47.6, 42.6, 36.6, 31.1, 25.9; IR (ATR): $v_{\max } 2926,1510,1260,1214,1117,1099,762,641$ $\mathrm{cm}^{-1} ;$ ESIHRMS m/z calcd $\mathrm{C}_{38} \mathrm{H}_{43} \mathrm{~N}_{2} \mathrm{O}_{6}[\mathrm{M}+\mathrm{H}]^{+}$623.3116, found 623.3116 . 


\section{Assignment of the Absolute Configuration of $(R)-12$ :}

The absolute configuration of $(R)-\mathbf{1 2}$ was assigned by chemical correlation with previously reported (R)-6,7-dimethoxy-1-(4-methoxyphenethyl)-1,2,3,4-tetrahydroisoquinoline ${ }^{54}$ according to the following scheme. Only the final intermediate - whose spectral data fully matched the ones previously reported ${ }^{54}$ - was fully characterized.

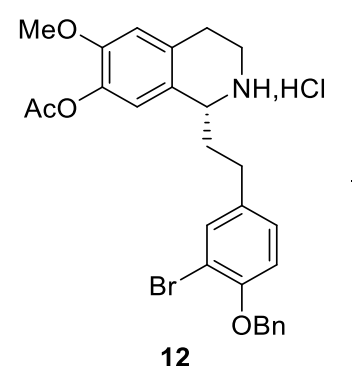

12
1. $\mathrm{H}_{2}, \mathrm{Pd} / \mathrm{C}, \mathrm{Et}_{3} \mathrm{~N}$.

$$
\underset{\text { 2. } \mathrm{K}_{2} \mathrm{CO}_{3}, \mathrm{MeOH}, \mathrm{rt}}{\stackrel{\mathrm{MeOH} / \mathrm{DCM}, \mathrm{rt}}{\longrightarrow}}
$$

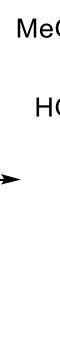

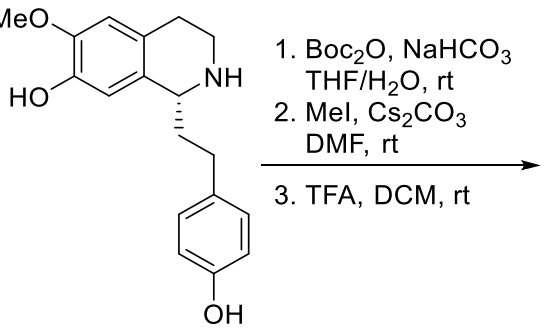

$$
\begin{aligned}
& {[\alpha]_{D}^{23}=+15(c) 0.6,}
\end{aligned}
$$



$$
\begin{aligned}
& \mathrm{CHCl}_{3} \text { ) }
\end{aligned}
$$

\section{(R)-6,7-Dimethoxy-1-(4-methoxyphenethyl)-1,2,3,4-tetrahydroisoquinoline. ${ }^{\mathrm{S}}$}

To a solution of 12 (310 mg, $0.57 \mathrm{mmol})$ in a mixture of $\mathrm{MeOH} / \mathrm{CH}_{2} \mathrm{Cl}_{2}(1 / 1,30 \mathrm{~mL})$ was added triethylamine dropwise $(87 \mu \mathrm{l}, 0.6 \mathrm{mmol})$ at $0{ }^{\circ} \mathrm{C}$. The reaction mixture was stirred 10 minutes at room temperature before adding palladium on carbon (10\% wt., $126 \mathrm{mg})$. The reaction mixture was stirred at room temperature under an hydrogen atmosphere (3.0 bars) for 24 hours. The reaction mixture was filtered through a pad of Celite ${ }^{\circledR}$ and the filtrate was concentrated under reduced pressure. The residue was then diluted with dichloromethane and successively washed with a saturated aqueous solution of sodium bicarbonate and brine, dried over magnesium sulfate, filtered and concentrated under reduced pressure.

The residue was dissolved in methanol $(5 \mathrm{~mL})$, treated with potassium carbonate $(1.2 \mathrm{~g}$, $8.5 \mathrm{mmol}$ ) and stirred overnight. The reaction mixture was then quenched by a slow addition of a $10 \%$ solution of $\mathrm{HCl}$ until pH 8 and extracted with dichloromethane. The combined organic layers were washed with brine, dried over magnesium sulfate and concentrated under reduced




pressure. The residue was purified by flash chromatography over silica gel (chloroform/methanol/triethylamine: $10 / 1 / 0.12)$ to give the intermediate $(R)$-1-(4hydroxyphenethyl)-6-methoxy-1,2,3,4-tetrahydroisoquinolin-7-ol (105 mg, $0.35 \mathrm{mmol}, 62 \%$ over two steps).

To a solution of this intermediate (R)-1-(4-hydroxyphenethyl)-6-methoxy-1,2,3,4tetrahydroisoquinolin-7-ol (105 mg, $0.35 \mathrm{mmol}$ ) in a mixture of $\mathrm{H}_{2} \mathrm{O} / \mathrm{THF}(2 / 1,5 \mathrm{~mL})$ was added sodium bicarbonate $(59 \mathrm{mg}, 0.7 \mathrm{mmol}$ ) and di-tert-butyl dicarbonate $(93 \mathrm{mg}, 0.43 \mathrm{mmol})$. The reaction mixture was stirred overnight, diluted with water and extracted with dichloromethane. The combined organic layers were successively washed with an $1 \mathrm{M}$ aqueous solution of $\mathrm{HCl}$ and brine, dried over magnesium sulfate and concentrated under reduced pressure. The residue was purified by flash chromatography over silica gel (petroleum ether/ ethyl acetate: $1 / 1)$ to give the intermediate $(R)-N$-tert-butoxycarbonyl-1-(4hydroxyphenethyl)-6-methoxy-1,2,3,4-tetrahydroisoquinolin-7-ol (110 mg, 0.275 mmol, 79\%).

To a solution of this intermediate (R)-N-tert-butoxycarbonyl-1-(4-hydroxyphenethyl)-6methoxy-1,2,3,4-tetrahydroisoquinolin-7-ol (110 mg, $0.275 \mathrm{mmol})$ in dry DMF (4 mL) was added cesium carbonate $(717 \mathrm{mg}, 2.2 \mathrm{mmol}$ ) and iodomethane $(52 \mu \mathrm{l}, 0.826 \mathrm{mmol})$ at room temperature. The reaction mixture was stirred overnight at room temperature, diluted with water and extracted with dichloromethane. The combined organic layers were washed with brine, dried over magnesium sulfate, filtered and concentrated under reduced pressure. The residue was purified by flash chromatography over silica gel (petroleum ether/ethyl acetate: $3 / 1)$ to give the intermediate $(R)-N$-tert-butoxycarbonyl-6,7-dimethoxy-1-(4methoxyphenethyl)-1,2,3,4-tetrahydroisoquinoline (75 mg, $0.175 \mathrm{mmol}, 64 \%$ ).

To the solution of this intermediate $(R)$ - $N$-tert-butoxycarbonyl-6,7-dimethoxy-1-(4methoxyphenethyl)-1,2,3,4-tetrahydroisoquinoline in dichloromethane (4 mL) was added dropwise trifluoroacetic acid ( $354 \mu \mathrm{L}, 4.63 \mathrm{mmol})$ at room temperature. The reaction mixture was stirred for three hours at room temperature and then poured into a saturated aqueous solution of sodium bicarbonate. The aqueous layer was extracted with dichloromethane and the combined organic layers were washed with brine, dried over magnesium sulfate, filtered and concentrated under reduced pressure to give the desired (R)-6,7-dimethoxy-1-(4methoxyphenethyl)-1,2,3,4-tetrahydroisoquinoline (44 mg, 77\%). $[\alpha]_{\mathrm{D}}^{23}+15\left(\mathrm{c} 0.6, \mathrm{CHCl}_{3}\right)\left(\right.$ lit. $^{\mathrm{S4}}$ : 
$[\alpha]_{\mathrm{D}}^{20}+14\left(c 0.5, \mathrm{CHCl}_{3}\right) ;{ }^{1} \mathrm{H} N M R\left(\mathrm{CDCl}_{3}, 300 \mathrm{MHz}\right): \delta 7.15(\mathrm{~d}, J=8.7 \mathrm{~Hz}, 2 \mathrm{H}), 6.84(\mathrm{~d}, J=8.7 \mathrm{~Hz}$, $2 \mathrm{H}), 6.57(\mathrm{~s}, 2 \mathrm{H}), 3.97-3.93(\mathrm{~m}, 1 \mathrm{H}), 3.85(\mathrm{~s}, 3 \mathrm{H}), 3.83(\mathrm{~s}, 3 \mathrm{H}), 3.79(\mathrm{~s}, 3 \mathrm{H}), 3.29-3.21(\mathrm{~m}, 1 \mathrm{H})$, 3.04-2.95 (m, $1 \mathrm{H}), 2.84-2.63(\mathrm{~m}, 4 \mathrm{H}), 2.16-1.96(\mathrm{~m}, 2 \mathrm{H}) ;{ }^{13} \mathrm{C} \mathrm{NMR}\left(\mathrm{CDCl}_{3}, 75 \mathrm{MHz}\right): \delta$ 157.9, 147.4 (2C), 134.5, 131.4, 129.4, 127.4, 114.0, 112.0, 109.3, 56.2, 56.0, 55.4, 55.2, 41.2, 38.6, $31.7,29.6$. 
Supporting Information

\section{${ }^{1} \mathrm{H}$ and ${ }^{13} \mathrm{C}$ NMR spectra}


<smiles>O=C(O)CCc1ccc(O)c(Br)c1</smiles>
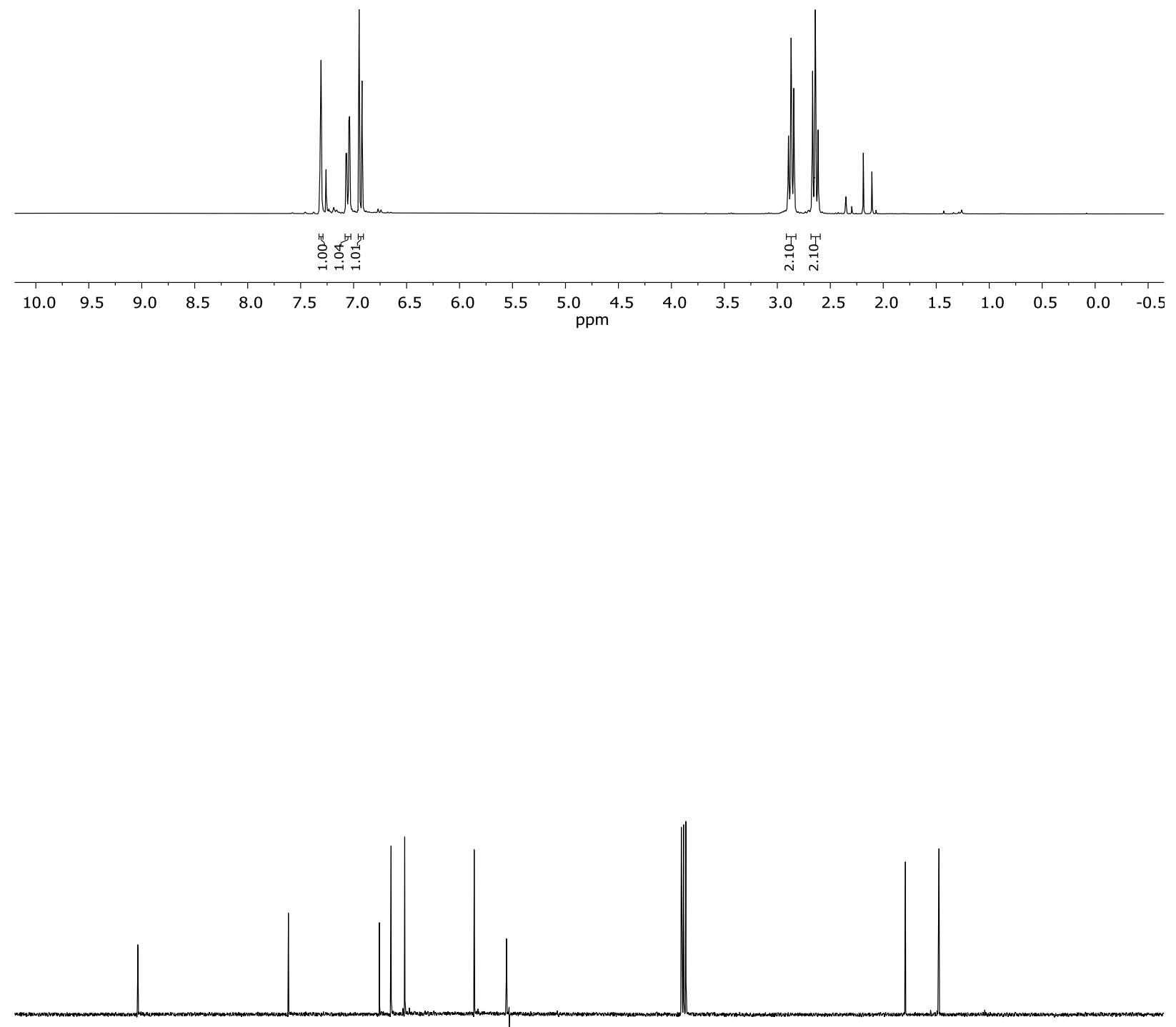

$\begin{array}{lllllllllllllllllllllll}200 & 190 & 180 & 170 & 160 & 150 & 140 & 130 & 120 & 110 & 100 & 90 & 80 & 70 & 60 & 50 & 40 & 30 & 20 & 10 & 0 & -10\end{array}$ 
<smiles>O=C(O)CCc1ccc(OCc2ccccc2)c(Br)c1</smiles>
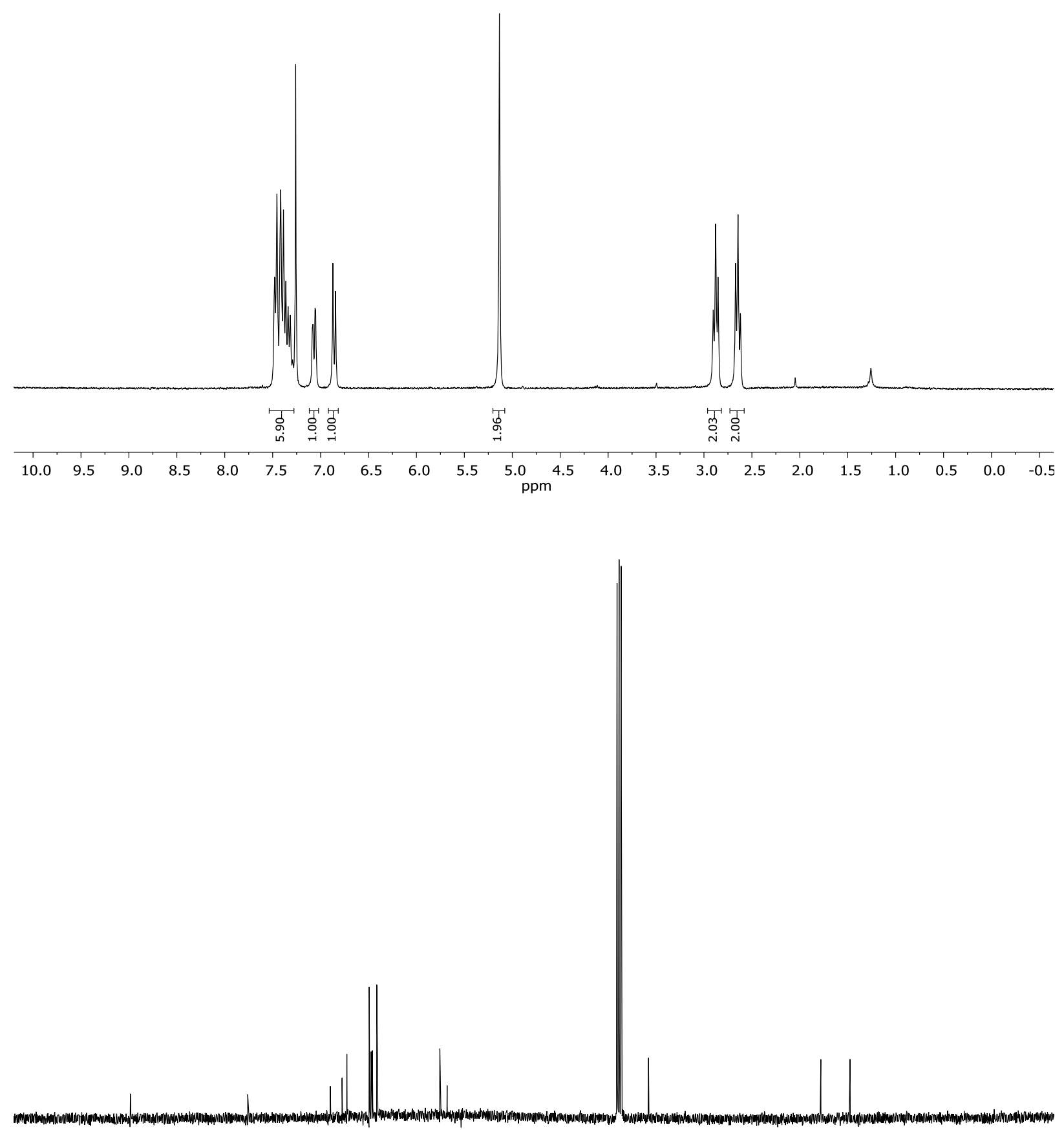

$\begin{array}{lllllllllllllllllllllll}200 & 190 & 180 & 170 & 160 & 150 & 140 & 130 & 120 & 110 & 100 & 90 & 80 & 70 & 60 & 50 & 40 & 30 & 20 & 10 & 0 & -10\end{array}$ 



$\begin{array}{lllllllllllllllllllllllllll}210 & 200 & 190 & 180 & 170 & 160 & 150 & 140 & 130 & 120 & 110 & 100 & 90 & 80 & 70 & 60 & 50 & 40 & 30 & 20 & 10 & 0 & -10\end{array}$ 



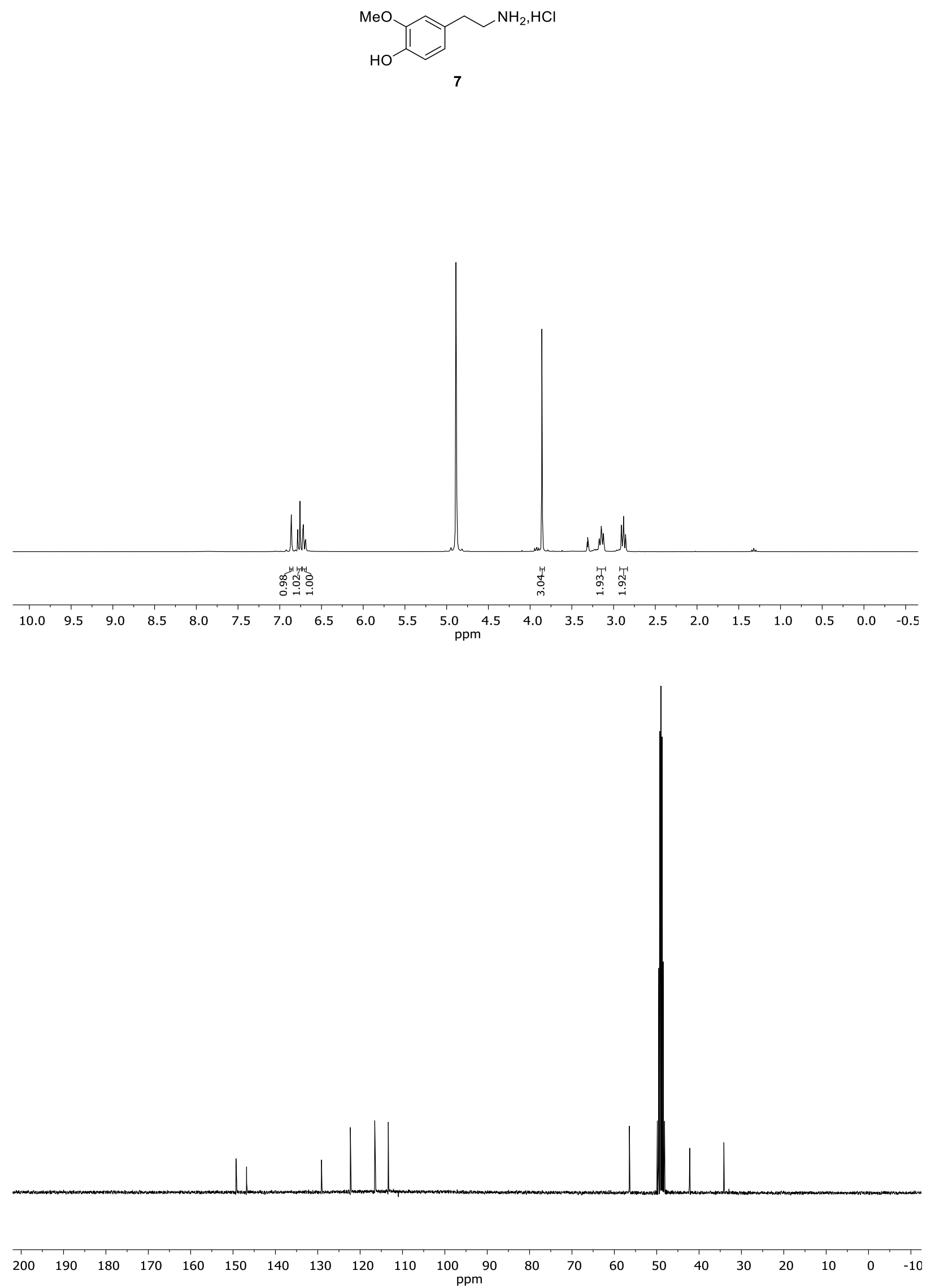

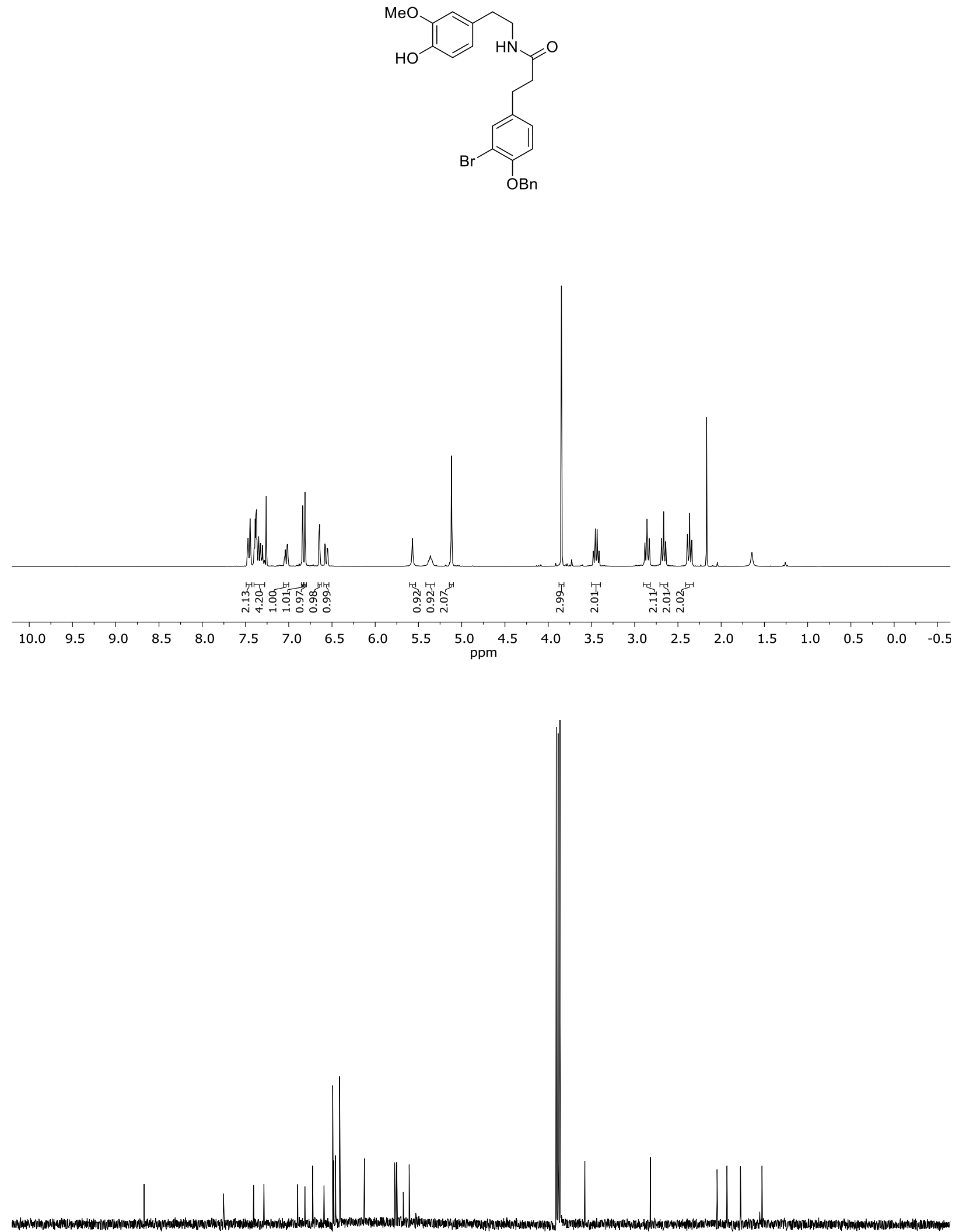

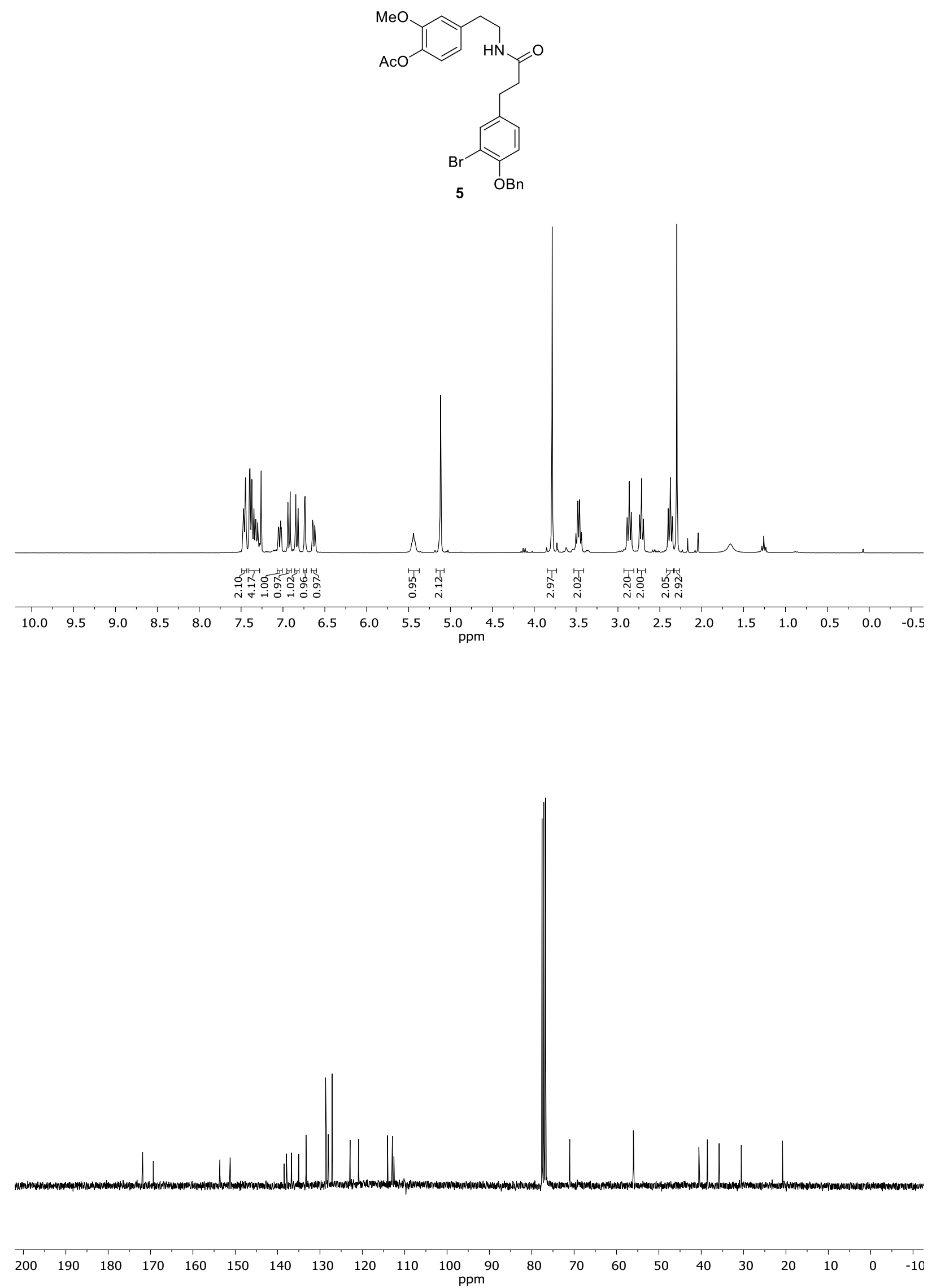



$\begin{array}{llllllllllllllllllllll}200 & 190 & 180 & 170 & 160 & 150 & 140 & 130 & 120 & 110 & 100 & 90 & 80 & 70 & 60 & 50 & 40 & 30 & 20 & 10 & 0 & -10\end{array}$ 

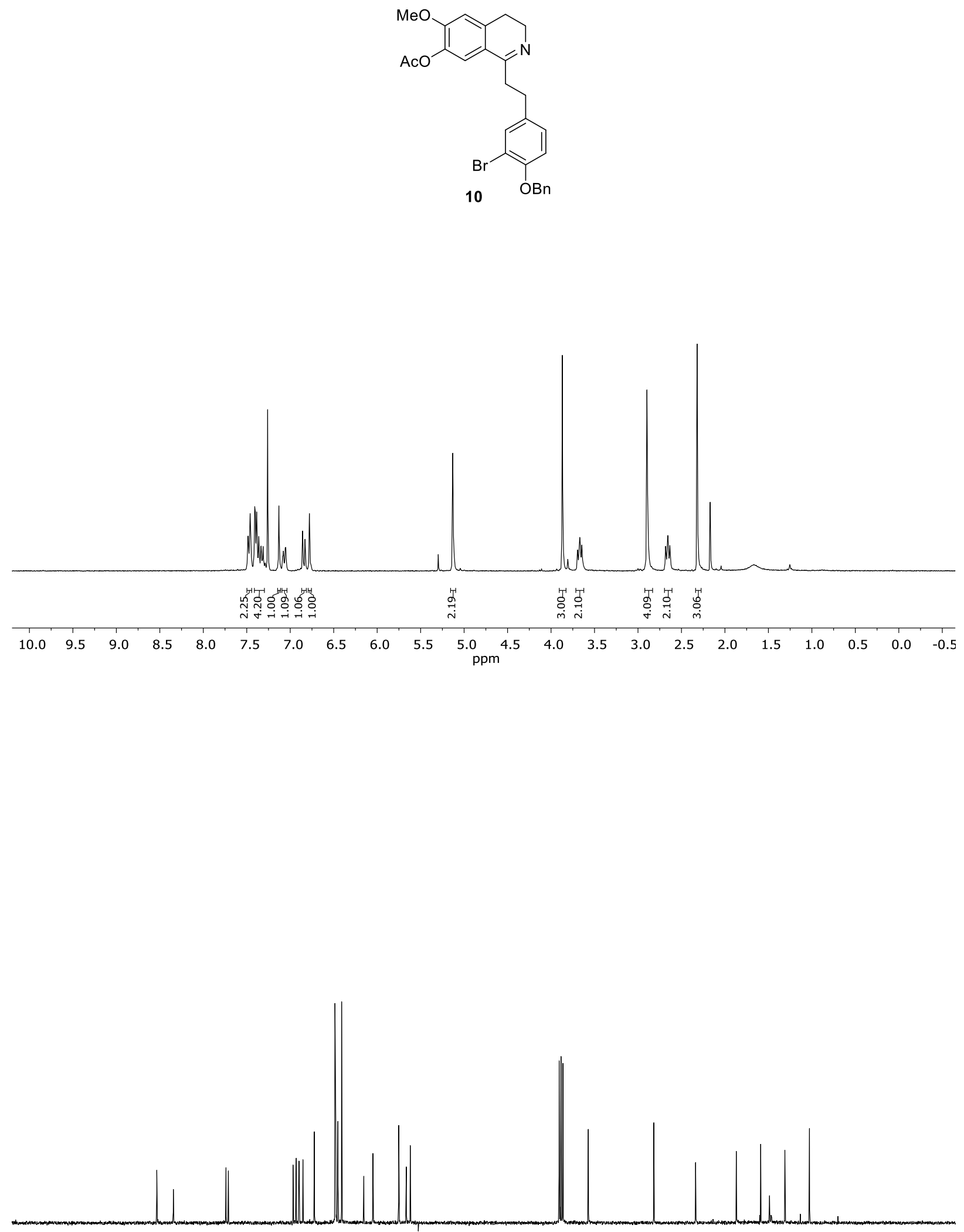
Total Synthesis of (-)-Melanthioidine by Copper-Mediated Cyclodimerization
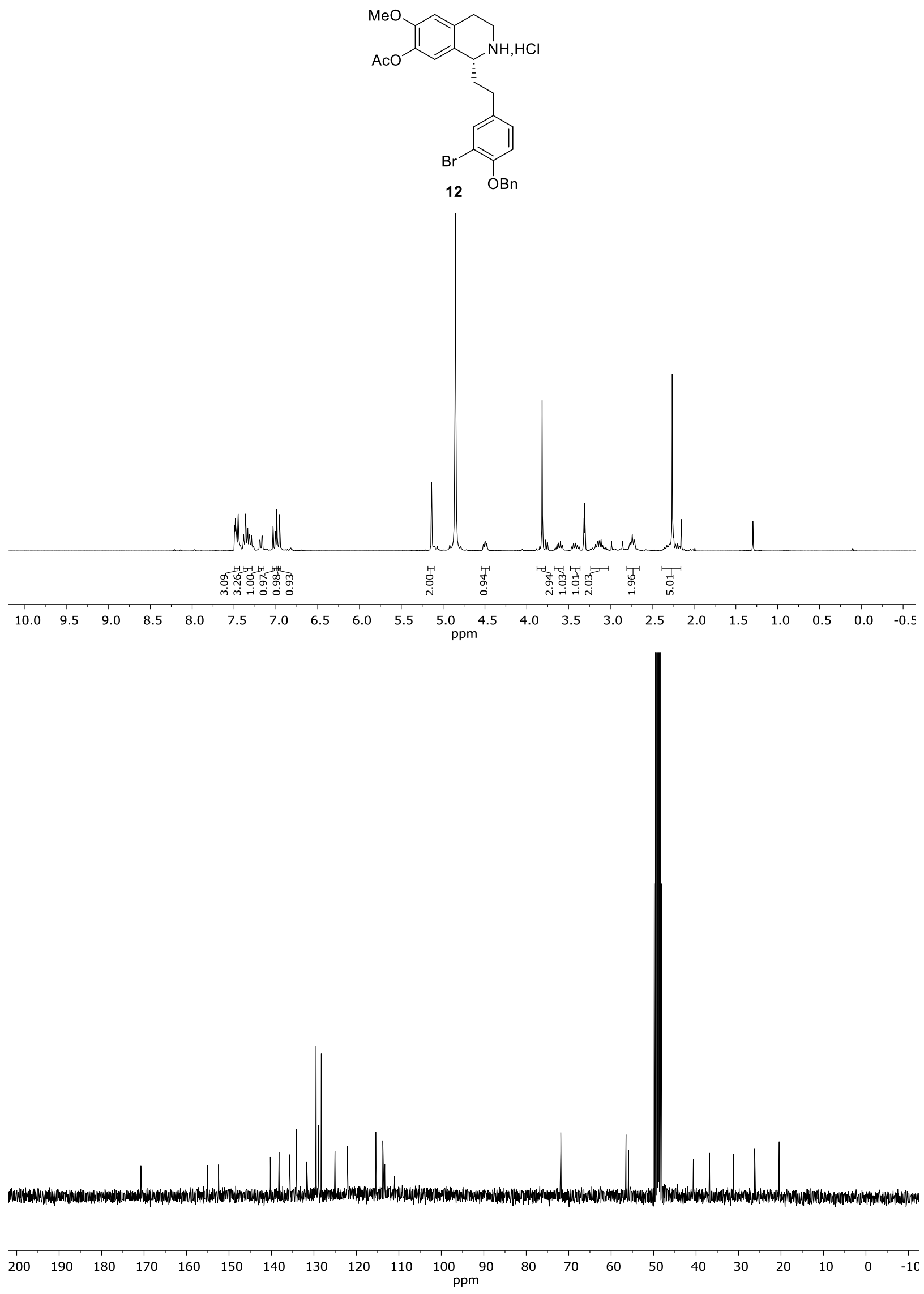

S27 

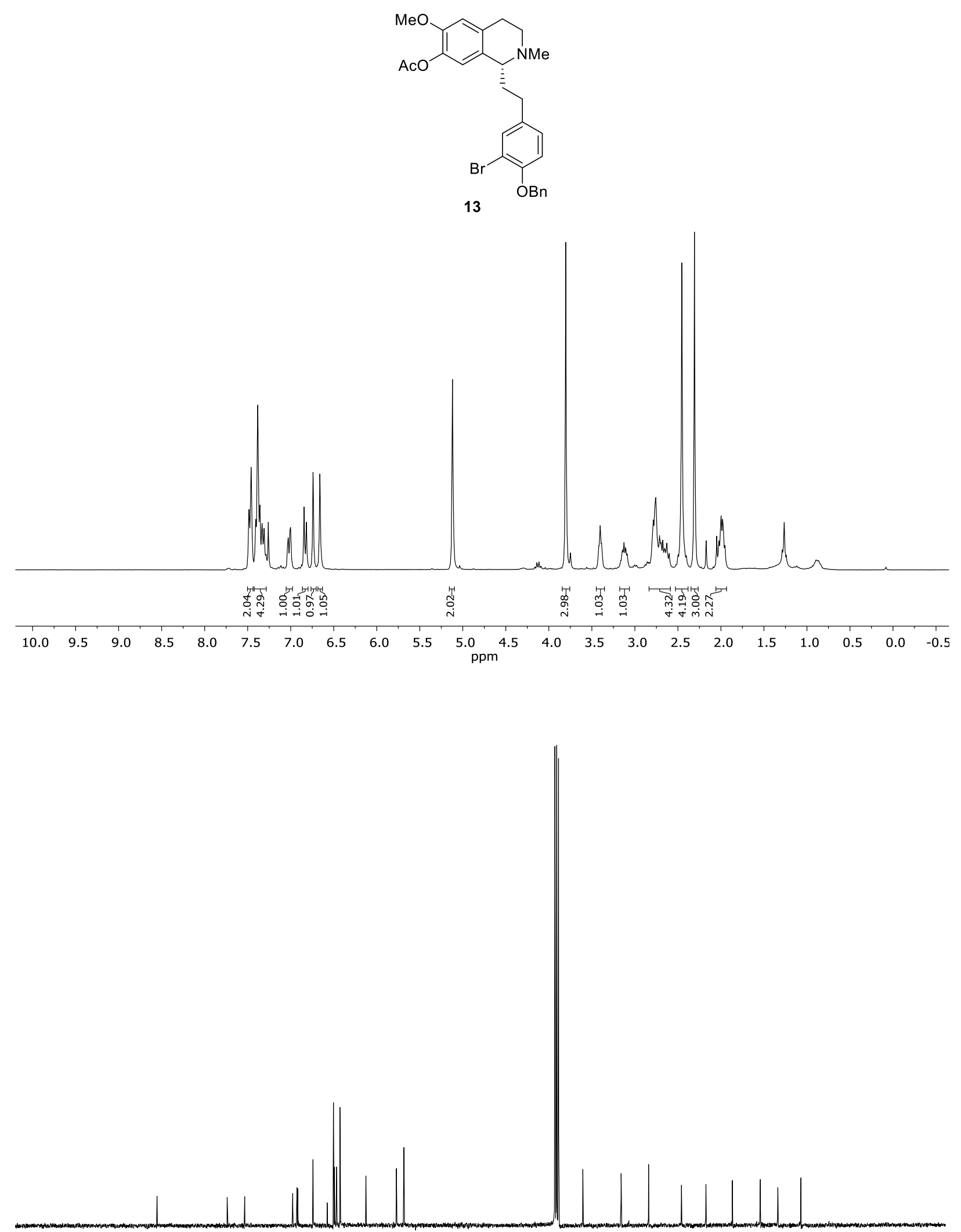

\begin{tabular}{llllllllllllllllllllll}
\hline 200 & 190 & 180 & 170 & 160 & 150 & 140 & 130 & 120 & 110 & 100 & 90 & 80 & 70 & 60 & 50 & 40 & 30 & 20 & 10 & 0 & -10
\end{tabular} 



$\begin{array}{llllllllllllllllllllllllll}200 & 190 & 180 & 170 & 160 & 150 & 140 & 130 & 120 & 110 & 100 & 90 & 80 & 70 & 60 & 50 & 40 & 30 & 20 & 10 & 0 & -10\end{array}$ 



\begin{tabular}{llllllllllllllllllllllll}
\hline 200 & 190 & 180 & 170 & 160 & 150 & 140 & 130 & 120 & 110 & 100 & 90 & 80 & 70 & 60 & 50 & 40 & 30 & 20 & 10 & 0 & -10
\end{tabular} 

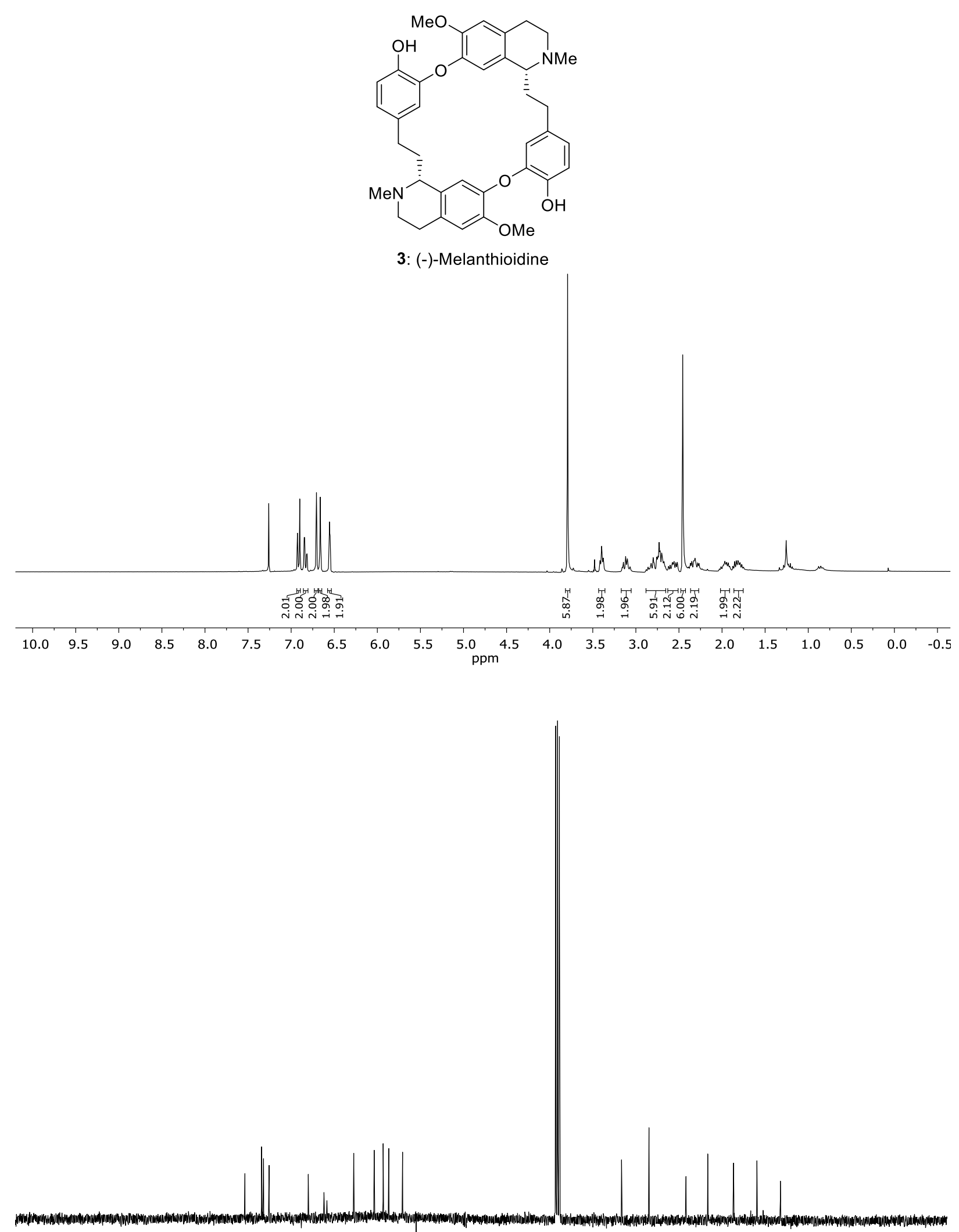

\begin{tabular}{llllllllllllllllllllllll}
\hline 200 & 190 & 180 & 170 & 160 & 150 & 140 & 130 & 120 & 110 & 100 & 90 & 80 & 70 & 60 & 50 & 40 & 30 & 20 & 10 & 0 & -10
\end{tabular} 

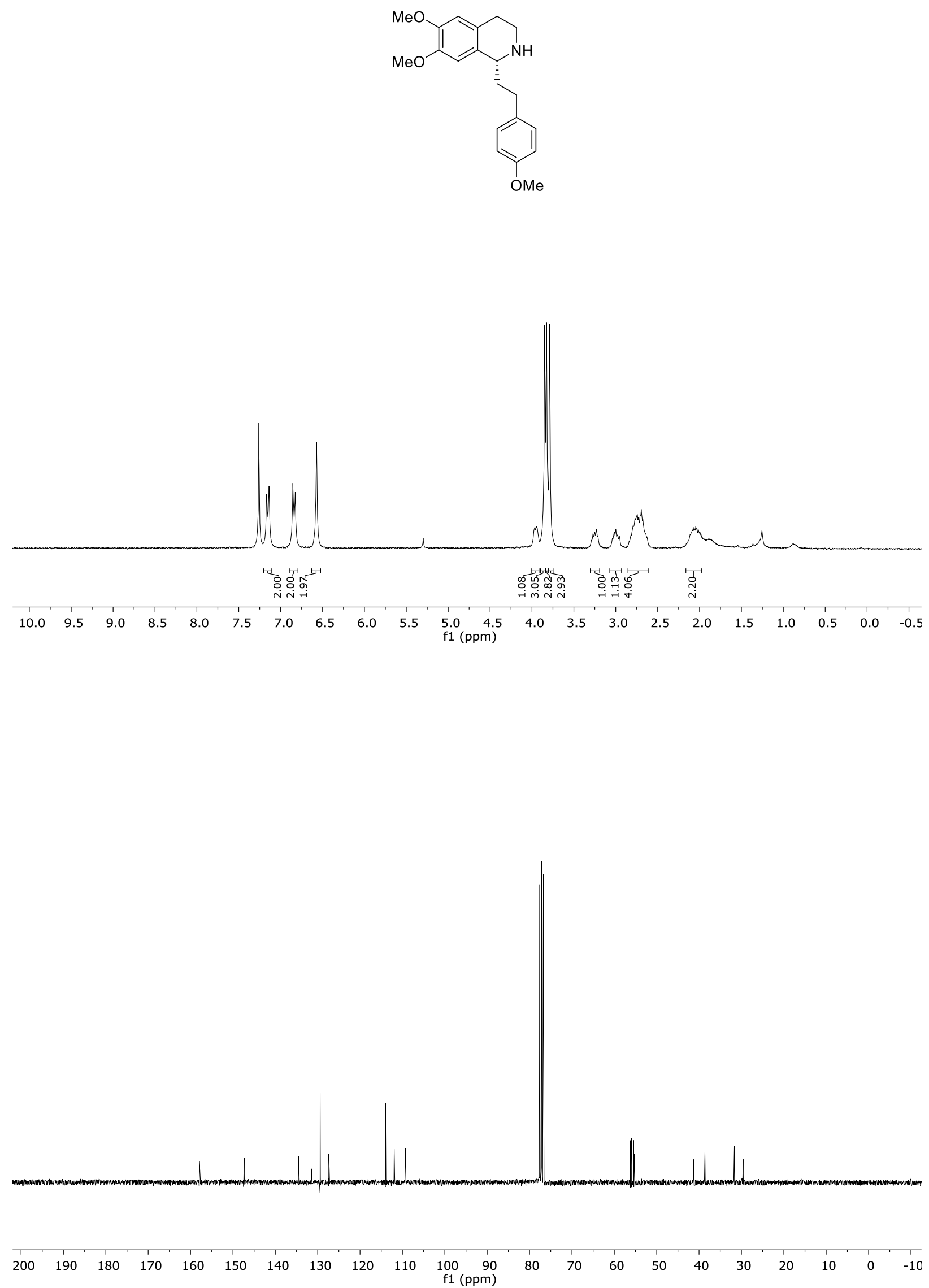
Supporting Information

Chiral HPLC Analysis of $(R)-13$ 


\section{Instrument method:}

Daicel Chiralpak IA-3 $(0.46 \mathrm{~cm} \phi \times 15 \mathrm{~cm}), 1.0 \mathrm{~mL} / \mathrm{min}$, room temperature 5\% Propan-2-ol 95\% $n$-hexane

Processed Channel PDA 200-600 nm

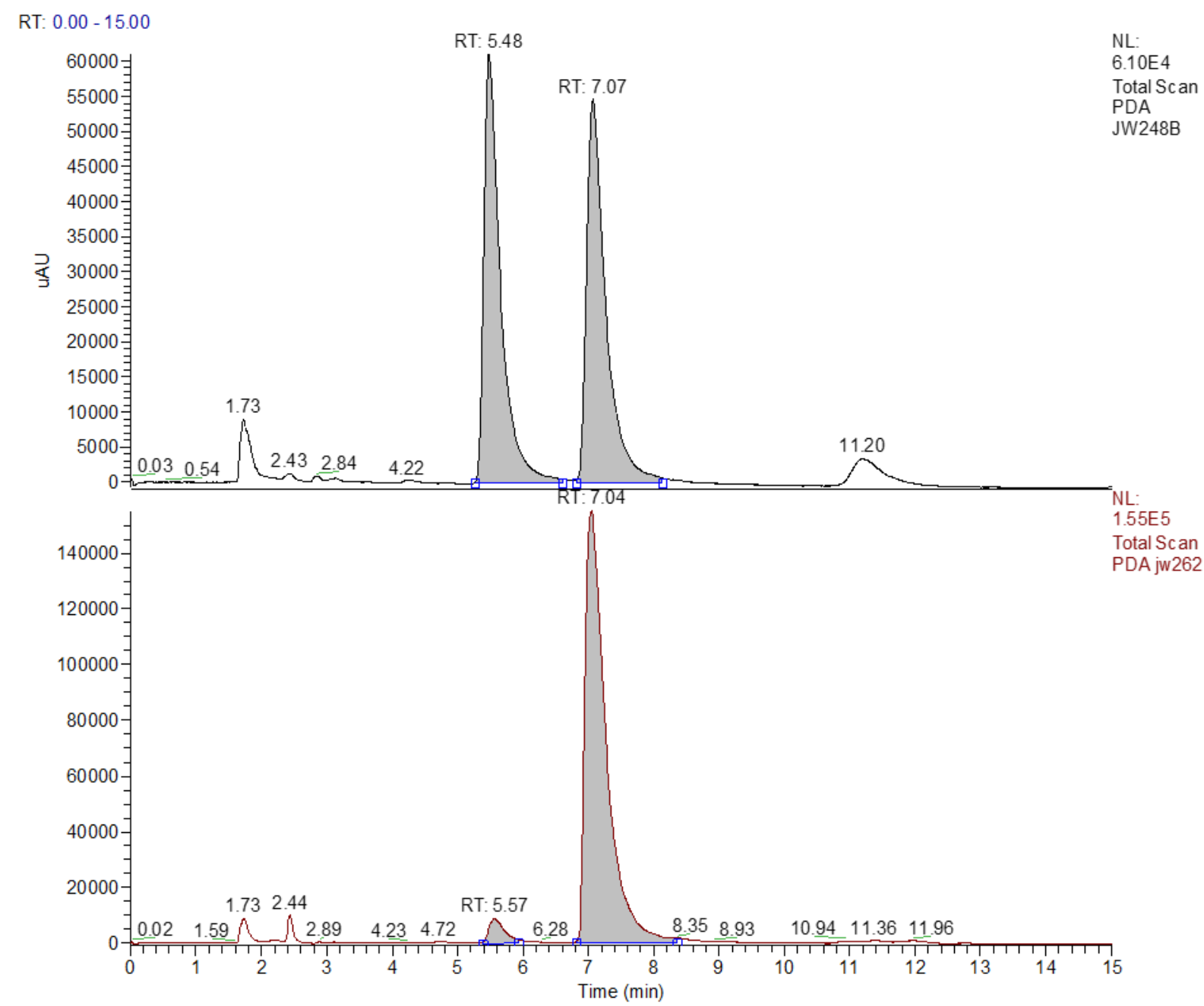

\section{Peak Results}

\begin{tabular}{|l|l|c|c|c|}
\hline & name & $\begin{array}{c}\text { Retention Time } \\
(\min )\end{array}$ & Area & \% Area \\
\hline 1 & Peak 1 & 5.57 & 158064.675 & 4.32 \\
\hline 2 & Peak 2 & 7.04 & 3497459.398 & 95.68 \\
\hline
\end{tabular}

